\title{
O impacto na qualidade de vida de cuidadores inseridos em um ambiente de terapia grupal para afásicos
}

\section{The impact on the quality of life of caregivers inserted in an aphasia group environment therapy}

Roxele Ribeiro Lima' (1), Natalia Cristina Silveira ${ }^{1}$ (1), Helbert do Nascimento Lima ${ }^{2}$ (1)

\begin{abstract}
RESUMO
Objetivo: o presente estudo comparou a qualidade de vida e percepções de cuidadores de sujeitos afásicos inseridos em um contexto de terapia grupal para afásicos. Métodos: trata-se de um estudo transversal, observacional e quantitativo, realizado com 13 cuidadores de indivíduos afásicos que receberam atendimento grupal e 13 cuidadores de sujeitos afásicos que não receberam atendimento fonoaudiólogo grupal. Para coleta dos dados, foram realizadas entrevistas individuais com os cuidadores e utilizou-se o Questionário de Sobrecarga do Cuidador (Burden Interview - Zarit). Resultados: constatouse que $45,2 \%$ dos cuidadores referiram sobrecarga de moderada a severa. No entanto, a sobrecarga avaliada por meio do questionário foi de leve a moderada e sem diferença significativa entre o grupo com e sem terapia fonoaudiológica. Conclusão: existe importante impacto na qualidade de vida de cuidadores de pacientes com afasia. Novos estudos são necessários para aprofundamento do papel da terapia fonoaudiológica em grupo na qualidade de vida de cuidadores.
\end{abstract}

Palavras-chave: Acidente vascular cerebral; Afasia; Cuidador; Qualidade de vida; Saúde de grupos específicos

\begin{abstract}
Purpose: The present study compared the quality of life and the perception of caregivers of aphasics in a context of group therapy for people with aphasia. Methods: This is a cross-sectional, observational, and quantitative study, carried out with 13 aphasic caregivers who received group care and 13 aphasic caregivers who did not receive a group speech therapist. For data collection, individual interviews with caregivers were carried out, using the Burden Interview - Zarit. Results: It was found that $45.2 \%$ of caregivers reported moderate to severe burden. However, the burden assessed by means of the questionnaire was mild to moderate and there was no significant difference between the group with and without speech therapy. Conclusion: There is an important impact on the quality of life of caregivers of patients with aphasia. Further studies are needed to deepen the role of group speech therapy in the quality of life of caregivers.
\end{abstract}

Keywords: Stroke; Aphasia; Caregiver; Quality of life; Health of specific groups

Trabalho realizado na Associação Educacional Luterana Bom Jesus, Faculdade IELUSC - Joinville (SC) Brasil.

${ }^{1}$ Departamento de Fonoaudiologia, Associação Educacional Luterana Bom Jesus, Faculdade IELUSC - Joinville (SC), Brasil.

${ }^{2}$ Departamento de Medicina, Universidade da Região de Joinville - UNIVILLE - Joinville (SC), Brasil.

Conflito de interesses: Não.

Contribuição dos autores: RRL participou da elaboração, desenvolvimento e escrita do manuscrito; NCS realizou a coleta, desenvolvimento e escrita do manuscrito; HNL colaborou no desenvolvimento, análise de dados e revisão do manuscrito.

Financiamento: Nada a declarar.

Autor correspondente: Roxele Ribeiro Lima. E-mail: roxele.lima@ielusc.br

Recebido: Abril 08, 2021; Aceito: Junho 03, 2021 


\section{INTRODUÇÃO}

Cerca de um terço dos pacientes com acidente vascular cerebral apresenta afasia ${ }^{(1)}$. A afasia é caracterizada por uma dificuldade de comunicação, que pode alterar o convívio familiar e social, bem como diminuição na qualidade de vida do sujeito afásico ${ }^{(2)}$ e de seu cuidador ${ }^{(3)}$. A terapia grupal (TG) para pacientes com afasia tem sido amplamente utilizada ${ }^{(4)} \mathrm{e}$, por sua característica de interação e socialização, possibilita que tais sujeitos estejam inseridos em um contexto de conversação ${ }^{(5,6)}$. No entanto, ainda pouco se sabe sobre a influência na TG na melhora da qualidade de vida de cuidadores de pacientes com afasia, envolvidos nesse contexto terapêutico.

Os pacientes com afasia frequentemente enfrentam sofrimento emocional em razão das limitações comunicativas ${ }^{(7)}$, com tendência para apresentar ansiedade, solidão e frustração ${ }^{(8,9)}$. Tais situações acarretam uma participação social mais restrita, com casos de depressão e mudanças no humor ${ }^{(10)}$, bem como menor retorno ao trabalho ${ }^{(11)}$. Com isso, os efeitos da afasia podem causar significativas mudanças na qualidade de vida dos sujeitos afetados ${ }^{(7)}$ e também nos seus cuidadores. No que se refere ao impacto da afasia na vida dos cuidadores, sabese que eles estão mais propensos a desenvolver depressão e experimentar uma variedade de consequências psicossociais após o início do convívio com alguém com afasia ${ }^{(12)}$. Além disso, o ato de cuidar gera diminuição do tempo e energia para suas próprias atividades, como aquelas sociais habituais ${ }^{(13)}$, resultando em isolamento social ${ }^{(14)}$.

Os cuidadores são considerados indivíduos que têm a função de auxiliar e/ou realizar a atenção adequada às pessoas que apresentam limitações para as atividades básicas da vida diária, estimulando a independência e respeitando a autonomia destas $^{(15)}$. O processo de cuidar é marcado por cansaço, estresse e esgotamento, o que coloca a família cuidadora na posição de necessitar, ela própria, de cuidado e de atenção ${ }^{(12)}$. É na fase de reabilitação que os familiares, cuidadores e o paciente experimentam totalmente o impacto do AVC, pois as mudanças ocasionadas no estilo de vida dos indivíduos afásicos refletem na vida dos seus cuidadores ${ }^{(16)}$.

O contato com um grupo terapêutico, tanto para os afásicos, quanto para seus cuidadores, é uma forma eficaz de interação $0^{(6,17)}$, pois o processo terapêutico grupal permite que os sujeitos despertem para assuntos desconhecidos e/ou inconscientes, discutam, reflitam e transfiram para a vida conhecimentos importantíssimos que os auxiliarão a serem agentes de sua própria saúde, durante ou após o período de terapia ${ }^{(17,18)}$. Com essa interação e o apoio que ocorrem em um grupo, pressupõe-se que haja melhor qualidade de vida para o cuidador e para os pacientes afásicos, após atendimento grupal ${ }^{(5)}$. Além de potencializar a qualidade de vida desses cuidadores, por meio da TG eles podem aumentar sua compreensão sobre AVC e afasia, favorecer um maior contato com outros cuidadores e a troca de experiências e vivências.

Considerando o número reduzido de estudos relacionados ao impacto da terapia grupal na qualidade de vida dos cuidadores, existe a necessidade de ampliar o conhecimento sobre as implicações da dinâmica da TG para sujeitos afásicos aos seus cuidadores. Assim, o presente estudo buscou avaliar o impacto na qualidade de vida de cuidadores de sujeitos afásicos que estão inseridos em um contexto de terapia grupal.

\section{MÉTODO}

Trata-se de um estudo transversal e observacional com o uso de uma entrevista semiestruturada e aplicação do Questionário de Sobrecarga do Cuidador (Burden Interview - Zarit) ${ }^{(19)}$. Os participantes foram selecionados por processo de amostragem não probabilística de conveniência.

Foram incluídos neste estudo cuidadores de pacientes afásicos fluentes e não fluentes, que frequentaram um grupo de terapia fonoaudiológica há pelo menos três meses, na clínica-escola da Associação Educacional Luterana Bom Jesus/IELUSC - Faculdade IELUSC, Joinville (SC). O grupo comparativo foi formado por cuidadores de indivíduos afásicos que ainda não tinham iniciado terapia fonoaudiológica grupal. Todos os pacientes afásicos incluídos apresentavam severidade leve, com pontuação de 4 a 5 pelo Teste de Boston para Diagnóstico das Afasias Reduzido (TBDA-R) ${ }^{(20)}$. Todos os cuidadores também eram residentes da cidade de Joinville (SC) e com idade igual ou superior a 18 anos.

Foram excluídos do estudo os participantes que não se enquadravam como cuidadores e/ou os cuidadores, cujos pacientes afásicos tinham outras doenças neurodegenerativas diagnosticadas.

Por meio da entrevista semiestruturada, foram analisadas as variáveis dos cuidadores, como idade, gênero, escolaridade, grau de parentesco e há quanto tempo desempenhavam a função de cuidador. Também foram coletados os dados dos participantes afásicos, como idade, gênero e tempo pós-AVC.

O Questionário de Sobrecarga do Cuidador (Burden Interview - Zarit) é um instrumento com 22 questões, com objetivo de avaliar a sobrecarga percebida pelo cuidador, e engloba as áreas de saúde, vida social e pessoal, situação financeira, bemestar emocional e relacionamento interpessoal. As questões são pontuadas numa escala de 0 a 4 pontos, representados da seguinte forma: nunca (0), raramente (1), algumas vezes (2), frequentemente (3) e sempre (4). A última questão do questionário refere-se, de maneira generalizada, à intensidade com a qual o cuidador se sente sobrecarregado por cuidar do paciente, sendo pontuada de 0 a 4, como: nem um pouco (0), um pouco (1), moderadamente (2), muito (3) e extremamente $(4)^{(19)}$. O questionário foi aplicado aos cuidadores dos dois grupos de pacientes com afasia: grupo com terapia (GT) e grupo sem terapia (GST).

A interação com o cuidador ocorreu durante o processo terapêutico grupal, que teve duração de 24 sessões (em média, três meses), duas vezes por semana, por um período de uma hora e 30 minutos. Nos 15 minutos finais de cada sessão, os cuidadores participavam de um momento de integração com os pacientes e terapeutas. Durante essa integração, eram fornecidas orientações sobre as atividades desenvolvidas durante a terapia, tarefas para serem realizadas em casa e estratégias ou técnicas para estabelecer uma comunicação eficaz entre o paciente com afasia e seu cuidador. Os cuidadores também participaram de dois grandes encontros, nos quais foram realizadas festas temáticas, além da terapia fonoaudiológica. Outro momento que uniu pacientes com afasia e cuidadores foi a escrita de um livro. No período de escrita, ambos tiveram que conversar, pensar e escrever, tendo, assim, um momento único para refletir sobre a vida pós-AVC, dificuldades, alegrias e mudança de visão de vida.

$\mathrm{O}$ estudo foi aprovado pelo Comitê de Ética em Pesquisa do IELUSC, sob protocolo 3.424.234 e todos os indivíduos, ou 
seus representantes legais, assinaram o Termo de Consentimento Livre e Esclarecido (TCLE).

\section{Análise estatística}

As variáveis qualitativas foram apresentadas pelo seu número absoluto e percentagem e as variáveis quantitativas, pela média e desvio padrão ou mediana e variação interquartil, conforme distribuição da variável. Foram utilizados os testes t Student ou Mann-Whitney U para comparar as variáveis quantitativas. Os testes Qui-quadrado, ou Exato de Fisher, foram utilizados para comparar as frequências de cada variável. Para realização das análises, foi utilizado o software SPSS, versão 23.

\section{RESULTADOS}

Dentre os 26 cuidadores participantes deste estudo, foi encontrada predominância do gênero feminino (80,8\%). Destas, 10 eram esposas dos pacientes, 9 eram filhas, 1 era mãe e, por fim, 1 era nora. Quanto aos homens (19,2\%), todos eram maridos de pacientes com afasia (Tabela 1).

A média geral de idade dos cuidadores entrevistados foi de 55,6 anos, sendo que $34,6 \%$ deles tinham entre 30 e 45 anos, $26,9 \%$, entre 46 e 60 anos e $38,4 \%$, acima de 60 anos.

Em relação ao nível de escolaridade houve prevalência de cuidadores que possuíam ensino médio $(65,4 \%)$, seguido de ensino fundamental completo $(19,2 \%)$.

Quanto aos pacientes com afasia, a média de idade foi de 67,3 anos e a gravidade da afasia foi de 4,6, grau leve, conforme classificação pelo TBDA- R, em que há desvantagens mínimas, como perda da fluência ou compreensão, mas que não são limitantes significativos para que o paciente expresse suas ideias. Da amostra geral, 57,7\% dos pacientes apresentavam algum comprometimento motor.

No que se refere ao tempo de cuidado prestado ao paciente afásico, a média foi de 8,2 meses, sendo que o tempo desempenhando a função de cuidador variou de 3 a 24 meses.

Quanto ao Questionário de Sobrecarga do Cuidador (Burden Interview - Zarit), a média geral da pontuação encontrada foi de 33,5 , classificada como sobrecarga leve a moderada ( 0 a 20 pontos $=$ ausência de sobrecarga; 21 a 40 pontos $=$ sobrecarga leve a moderada; 41 a 60 pontos = sobrecarga moderada a severa e acima de 61 pontos $=$ sobrecarga intensa).

Quando questionado aos cuidadores a respeito do quanto se sentiam sobrecarregados, $8(30,7 \%)$ relataram não sentir nenhuma sobrecarga, $6(23 \%)$, pouca sobrecarga, $7(26,1 \%)$, sobrecarga moderada, $4(15,3 \%)$, muita sobrecarga e apenas 1 cuidador se sentia extremamente sobrecarregado $(3,8 \%)$.

Na Tabela 2, estão expostas as variáveis entre os grupos (GT e GST). No que se refere ao gênero, em ambos os grupos foi demonstrada prevalência do gênero feminino ocupando o papel de cuidador. Com relação ao grau de escolaridade, ambos os grupos apresentaram maior índice de ensino médio completo $(\mathrm{GT}=69,2 \%$ e $\mathrm{GST}=61,5 \%)$.

Quando analisadas as características dos pacientes com afasia, verificou-se que a maioria dos acometidos era do gênero masculino, em ambos os grupos (GT $=53,8 \%$ e GST $=76,9 \%$, $p=0,411)$. Não houve diferença significativa no comprometimento motor entre os grupos $(\mathrm{p}=0,418)$.

Com relação ao Questionário de Sobrecarga do Cuidador (Burden Interview - Zarit), constatou-se que as médias foram semelhantes nos dois grupos, sendo encontrada no GT a média de 36,23 e, no GST, 30,84 ( $p=0,37$ ), ambos, portanto, classificados com sobrecarga leve a moderada. A média da gravidade da afasia foi semelhante nos grupos (4,5 no GT e 4,7 no GST, $p=0,584)$,

Tabela 1. Características gerais da amostra

\begin{tabular}{lcc}
\hline \multicolumn{1}{c}{ Variáveis } & Frequência ou média & Porcentagem ou Desvio Padrão \\
\hline Gênero do cuidador: & 5 & $19,20 \%$ \\
$\quad$ Masculino & 21 & $80,80 \%$ \\
Feminino & 55,6 & 13,5 \\
Idade do cuidador & & $7,70 \%$ \\
Nível de escolaridade do cuidador: & 2 & $19,20 \%$ \\
$\quad$ Analfabeto & 5 & $65,40 \%$ \\
Ensino fundamental & 17 & $7,70 \%$ \\
Ensino médio & 2 & 5,2 \\
Ensino superior & 8,2 & \\
Há quanto tempo é cuidador & & $65,40 \%$ \\
Gênero do afásico: & 17 & $34,60 \%$ \\
$\quad$ Masculino & 9 & 12,1 \\
Feminino & 67,3 & \\
Idade do afásico & & $42,30 \%$ \\
Comprometimento motor & 11 & $57,70 \%$ \\
$\quad$ Não & 15 & $50 \%$ \\
Sim & & $50 \%$ \\
Terapia grupal & 13 & 0,7 \\
Sim & 13 & 13,4 \\
Não & 4,6 & \\
Gravidade da afasia & 33,5 & \\
Questionário Sobrecarga do Cuidador & & \\
\hline
\end{tabular}


Tabela 2. Características gerais entre cuidadores com e sem terapia grupal fonoaudiológica $(n=26)$

\begin{tabular}{|c|c|c|c|c|c|}
\hline & \multicolumn{2}{|c|}{ Grupo com Terapia } & \multicolumn{2}{|c|}{ Grupo Sem Terapia } & \multirow[b]{2}{*}{ Valor de $\mathrm{p}$} \\
\hline & $\begin{array}{c}\text { Frequência } \\
\text { ou Média }\end{array}$ & DP & $\begin{array}{c}\text { Frequência } \\
\text { ou Média }\end{array}$ & DP & \\
\hline \multicolumn{6}{|l|}{ Gênero do cuidador } \\
\hline Feminino & $76,90 \%$ & & $84,60 \%$ & & \\
\hline Idade do cuidador & 56,8 & 12,8 & 54,5 & 14,6 & 0,638 \\
\hline Ensino Fundamental & $15,40 \%$ & & $23,10 \%$ & & ---- \\
\hline Ensino médio & $69,20 \%$ & & $61,50 \%$ & & \\
\hline Ensino superior & $0 \%$ & & $15,40 \%$ & & \\
\hline Tempo de cuidador & 10,4 & 6 & 6 & 3 & 0,034 \\
\hline Questionário & 36,2 & 12,6 & 30,8 & 14,1 & 0,317 \\
\hline Gravidade da afasia & 4,5 & 0,6 & 4,7 & 0,7 & 0,584 \\
\hline \multicolumn{6}{|c|}{ Comprometimento motor } \\
\hline Não & $30,80 \%$ & & $53,80 \%$ & & 0,418 \\
\hline Sim & $69,20 \%$ & & $46,20 \%$ & & \\
\hline
\end{tabular}

Legenda: $\mathrm{n}$ = número de sujeitos; $\mathrm{DP}=$ desvio padrão; esc. = escolaridade; Questionário = Questionário Sobrecarga do Cuidador

assim como a média de idade dos cuidadores ( 56,8 anos no GT e 54,5 anos no GST, $\mathrm{p}=0,683$ ).

Um dado importante na pesquisa foi o tempo de função de cuidador. No GT, a média foi de 10,4 meses, enquanto no GST, foi de 6 meses $(\mathrm{p}=0,034)$, constatando que, no GT, os cuidadores desempenhavam o papel de cuidador há mais tempo que os do GST.

\section{DISCUSSÃO}

O estudo explorou a sobrecarga dos cuidadores de pacientes com afasia e identificou que não houve diferença entre o GT e o GST. Todos os cuidadores apresentaram sobrecarga de leve a moderada e com maior prevalência do gênero feminino desempenhando o papel de cuidador. Além disso, o estudo apontou, de forma significativa, que o maior tempo de cuidador estava associado aos pacientes da amostra que realizaram terapia fonoaudiológica.

Sabe-se que ser cuidador informal significa uma mudança brusca para toda a família. Ter uma pessoa sobrevivente ao AVC em casa e que apresenta sequela motora e prejuízo na fala é um desafio. A afasia gera sobrecarga maior ao cuidador e essa dificuldade de comunicação pode abalar a relação do sujeito com a família, pois esta não consegue compreender o que o afásico está tentando expressar ${ }^{(21)}$. Com o comprometimento da comunicação gerado pela afasia e a sobrecarga do cuidador, faz-se necessário sua inserção em um contexto grupal que propicie interação com outros cuidadores, ressignificando seus sentimentos e trocas de experiências ${ }^{(22)}$.

Neste estudo, evidenciou-se que todos os cuidadores, independentemente de estarem, ou não, inseridos em um ambiente de terapia grupal, tiveram sobrecarga física e emocional de leve a moderada. Contudo, durante as entrevistas, mesmo o questionário contendo apenas respostas objetivas, os cuidadores que estavam inseridos no grupo relataram sobre sua rotina e dificuldades, o que não foi observado no GST. Muitos cuidadores do GT desabafaram sobre os seus anseios, as limitações com o trato do afásico e o impacto da afasia em sua vida. Alguns também relataram um senso maior de competência, realização de ganhos e melhor compreensão de sua situação. As cuidadoras esposas de pacientes referiram uma sensação de "alívio" e "esperança", quando comparavam a situação do companheiro com a de outros no grupo, ou porque eram mais velhos, porque que tinham mais tempo de AVC, ou por terem maior comprometimento motor, o que concorda com uma revisão realizada por Attard et al. (2013) que relata as falas dos cuidadores de forma semelhante. A angústia do cuidador verificada neste estudo comprova os achados de outros estudos da área ${ }^{(23,24)}$. Os cuidadores passam por mudanças bruscas de estilo de vida, tendo que modificar sua rotina e seus planos e, com isso, muitos necessitam deixar seus empregos ${ }^{(12)}$ e negligenciam o cuidado consigo mesmos, devido à sobrecarga emocional. Além disso, muitos deixam de lado seus desejos e vontades para cuidar de seu familiar ${ }^{(25)}$ necessitando que alguém fique com ele para que possam desfrutar de algumas atividades próprias ${ }^{(13)}$.

Sabe-se, também, que os cuidadores, quando inseridos em um ambiente de reabilitação, aumentam o seu interesse em entender mais sobre afasia e em como se comunicar melhor com o paciente ${ }^{(12)}$, podendo minimizar, assim, o estresse e os riscos de depressão que os cuidadores de afásicos apresentam ${ }^{(13,26)}$. Por conseguinte, percebe-se que muitas experiências adquiridas ao longo da vida são compartilhadas em um grupo de terapia fonoaudiológica ${ }^{(27)}$. Um grupo terapêutico é classificado como um conjunto de pessoas que partilham de um mesmo interesse e ou objetivo em comum e em que há interação e vínculo emocional pelos sujeitos envolvidos ${ }^{(28)}$. Estar inserido num contexto terapêutico propicia ao cuidador a troca de experiências 
de forma compartilhada, segurança ao perceber que não é o único a estar passando por aquela situação e também a troca de pensamentos positivos, o que pode contribuir para o seu bem-estar e melhora na sua qualidade de vida ${ }^{(27)}$.

Este estudo também evidenciou que a maioria dos cuidadores eram do gênero feminino, o que pode estar relacionado à representação sociocultural da mulher na sociedade, na qual a prática de cuidado é direcionada, geralmente, à esposa, devido ao compromisso matrimonial, ou à filha ${ }^{(21,24,29)}$, e isso está constituído dentro de uma esfera doméstica, o que confirma os dados obtidos no estudo.

De acordo com a escolaridade do cuidador, este possui relevância, visto que, após se tornar cuidador, é necessário que o busque informações e também receba orientações quanto ao cuidado com o paciente ${ }^{(29)}$, o que pode influenciar o processo de reabilitação do paciente, pois, quanto maior o grau de escolaridade, mais acesso a informações tem o cuidador ${ }^{(29)}$.

Este estudo apontou, também de forma significativa, que o maior tempo como cuidador estava associado aos pacientes que realizaram terapia fonoaudiológica. Considerando a limitação de vagas locais para o atendimento terapêutico grupal, indivíduos com mais tempo de afasia e, consequentemente, cuidadores com maior tempo de cuidado, podem representar, meramente, o tempo de espera para início de atendimento. No entanto, não se afasta a possibilidade de cuidadores com maior tempo de cuidado estarem mais engajados a essa nova rotina de cuidar e procurarem mais os serviços de reabilitação ${ }^{(30)}$.

Outro achado deste estudo foi que uma parte representativa dos pacientes apresentava sequela motora, com isso, pode-se inferir que pacientes, mesmo com limitação motora, buscam atendimento para sequelas de comunicação. Tal busca pode ser favorecida pela presença do próprio cuidador em compreender mais sobre a doença e a necessidade da reabilitação, auxiliando os pacientes na busca por atendimento fonoaudiólogico. Também é importante ressaltar que todos os pacientes com comprometimento motor fizeram terapia com fisioterapeuta, sugerindo que há uma valorização e um cuidado maior para estes pacientes por parte dos cuidadores.

As limitações deste estudo foram o número reduzido da amostra e a falta de maior vínculo com os cuidadores do GST, o que pode ter acarretado um viés de relato desse grupo. Sugerese, em futuros estudos, uma amostra maior e o acréscimo de perguntas abertas aos cuidadores, ampliando a visão sobre a sua sobrecarga. Entende-se que existe sobrecarga considerável na vida dos cuidadores de pacientes com afasia e o grupo terapêutico pode ser uma oportunidade de auxílio para esses indivíduos.

\section{CONCLUSÃO}

Este estudo demonstrou que há uma sobrecarga física e emocional de leve a moderada dos cuidadores de afásicos, em geral. Após se tornar cuidador, o indivíduo sofre com sobrecarga emocional, física e financeira, além de mudança no estilo de vida. Esses fatores, associados com a comunicação ineficaz, podem gerar um desgaste emocional maior para cuidadores de afásicos. Reforça-se a necessidade de um olhar para os cuidadores de pacientes com afasia, visando melhor qualidade em suas vidas. Por ser restrito o número cuidadores deste estudo, novos estudos são necessários para aprofundar e compreender melhor outros processos envolvidos nessa função e o papel da terapia grupal fonoaudiológica na qualidade de vida dos cuidadores.

\section{REFERÊNCIAS}

1. Lima RR, Rose ML, Lima HN, Cabral NL, Silveira NC, Massi GA. Prevalence of aphasia after stroke in a hospital population in southern Brazil: a retrospective cohort study. Top Stroke Rehabil. 2020 Abr;27(3):215-23. PMid:31687916.

2. Hilari K. The impact of stroke: are people with aphasia different to those without? Disabil Rehabil. 2011;33(3):211-8. http://dx.doi.org/ 10.3109/09638288.2010.508829. PMid:20712416.

3. Carleto NG, Caldana ML. Correlação entre a qualidade de vida do paciente afásico e de seu familiar. Distúrb Comun. 2014;26(4):630-40

4. Brady MC, Kelly H, Godwin J, Enderby P, Campbell P. Speech and language therapy for aphasia following stroke. Cochrane Database Syst Rev. 2016;2016(6):CD000425. http://dx.doi.org/10.1002/14651858. CD000425.pub4. PMid:27245310.

5. Lima RR, Rose ML, Lima HN, Guarinello AC, Santos RS, Massi GA. Socio-demographic factors associated with quality of life after a multicomponent aphasia group therapy in people with sub-acute and chronic post-stroke aphasia. Aphasiology. 2020;35(5): 642 -57.

6. Attard MC, Loupis Y, Togher L, Rose ML. The efficacy of an interdisciplinary community aphasia group for living well with aphasia. Aphasiology. 2018;32(2):105-38. http://dx.doi.org/10.1080/0268703 8.2017.1381877.

7. Hilari K, Needle JJ, Harrison KL. What are the important factors in health-related quality of life for people with aphasia? A systematic review. Arch Phys Med Rehabil. 2012;93(1):S86-95. http://dx.doi. org/10.1016/j.apmr.2011.05.028. PMid:22119074.

8. Cruice M, Worrall L, Hickson L. Health-related quality of life in people with aphasia: implications for fluency disorders quality of life research. J Fluency Disord. 2010;35(3):173-89. http://dx.doi. org/10.1016/j.jfludis.2010.05.008. PMid:20831966.

9. Dalemans RJ, Witte L, Wade D, van den Heuvel W. Social participation through the eyes of people with aphasia. Int J Lang Commun Disord. 2010;45(5):537-50. http://dx.doi.org/10.3109/13682820903223633. PMid:19839875.

10. Baker C, Worrall L, Rose M, Ryan B. 'It was really dark': the experiences and preferences of people with aphasia to manage mood changes and depression. Aphasiology. 2020;34(1):19-46. http://dx.doi.org/10.108 0/02687038.2019.1673304.

11. Simmons-Mackie N, Cherney L. Aphasia in North America: highlights of a white paper. Arch Phys Med Rehabil. 2018;99(10):e117. http:// dx.doi.org/10.1016/j.apmr.2018.07.417.

12. Grawburg M, Howe T, Worrall L, Scarinci N. Third-party disability in family members of people with aphasia: a systematic review. Disabil Rehabil. 2013;35(16):1324-41. http://dx.doi.org/10.3109/09638288. 2012.735341. PMid:23826903.

13. Jellema S, Wijnen MAM, Steultjens EMJ, Nijhuis-van der Sanden MWG, van der Sande R. Valued activities and informal caregiving in stroke: a scoping review. Disabil Rehabil. 2019;41(18):2223-34. http://dx.doi.org/10.1080/09638288.2018.1460625. PMid:29661038.

14. Costa TFD, Pimenta CJL, Nóbrega MMLD, Fernandes MDGM, França ISX, Pontes MLF, et al. Burden on caregivers of patients with sequelae of cerebrovascular accident. Rev Bras Enferm. 2020;73(6):e20180868. http://dx.doi.org/10.1590/0034-7167-2018-0868. PMid:32785520.

15. Nakatani AYK, Souto CCS, Paulette LM, Melo TS, Souza MM. Perfil dos cuidadores informais de idosos com déficit de autocuidado atendidos elo Programa de Saúde da Família. REE. 2003;5(1):15-20. 
16. Attard MC, Loupis Y, Togher L, Rose ML. Experiences of people with severe aphasia and spouses attending an Interdisciplinary Community Aphasia Group. Disabil Rehabil. 2020 Maio;42(10):1382-96. http:// dx.doi.org/10.1080/09638288.2018.1526336. PMid:30652928.

17. Attard MC, Lanyon L, Togher L, Rose ML. Consumer perspectives on community aphasia groups: a narrative literature review in the context of psychological well-being. Aphasiology. 2015;29(8):9831019. http://dx.doi.org/10.1080/02687038.2015.1016888.

18. Souza APR, Crestani AH, Vieira CR, Machado FCM, Pereira LL. O grupo na fonoaudiologia: origens clínicas e na saúde coletiva. Rev CEFAC. 2011;13(1):140-51.

19. Sequeira CAC. Adaptação e validação da Escala de Sobrecarga do Cuidador de Zarit. Revista de Enfermagem Referência. 2010;2(12):9-16.

20. Goodglass H, Kaplan E, Barresi B. The assessment of aphasia and related disorders. Philadelphia: Lippincott Williams \& Wilkins; 2001.

21. Panhoca I, Rodrigues N. Avaliação da qualidade de vida de cuidadores de afásicos. Rev Soc Bras Fonoaudiol. 2009 Nov; 14(3):394-401. http:// dx.doi.org/10.1590/S1516-80342009000300017.

22. Bernstein-Ellis E, Elman RJ. Aphasia group communication treatment: The Aphasia Center of California approach. In: Elman RJ, editor. Group treatment of neurogenic communication disorders: the expert clinician's approach. San Diego: Plural Pub; 2007. p. 71-94.

23. Grawburg M, Howe T, Worrall L, Scarinci N. Describing the impact of aphasia on close family members using the ICF framework. Disabil
Rehabil. 2014;36(14):1184-95. http://dx.doi.org/10.3109/09638288. 2013.834984. PMid:24063293.

24. Moleta F, Guarinello AC, Berberian AP, Santana AP. O cuidador familiar no contexto das afasias. Distúrb Comun. 2011;23(3):343-52.

25. Rodrigues SLA, Watanabe HAW, Derntl AM. A saúde de idosos que cuidam de idosos. Rev Esc Enferm USP. 2006;40(4):493-500. http:// dx.doi.org/10.1590/S0080-62342006000400007. PMid:17310565.

26. Silva F, Patrício B. Influência da comunicação na vida dos cuidadores de pessoas com afasia. In: I Congresso Internacional da Saúde GaiaPorto; Porto. Porto: Instituto Politécnico do Porto. Escola Superior de Tecnologia da Saúde do Porto - Politema; 2010.

27. Rizzolli D, Surdi AC. Percepção dos idosos sobre grupos de terceira idade. Rev Bras Geriatr Gerontol. 2010;13(2):225-33. http://dx.doi. org/10.1590/S1809-98232010000200007.

28. Zimerman D. A importância dos grupos na saúde, cultura e diversidade. Vínculo. 2007;4:1-16.

29. Ziesemer NBS, Lüders D, Gonçalves CGO, Hey AP, Guarinello AC, Massi G. Profile of professional home caregivers of senior citizens from a Southern Brazil setting. Research. Soc Dev. 2020;9(11):e59091110111.

30. Fonseca NR, Penna AFG, Soares MPG. Ser cuidador familiar: um estudo sobre as conseqüências de assumir este papel. Physis. 2008;18(4):72743. http://dx.doi.org/10.1590/S0103-73312008000400007. 